\title{
High Throughput Automatic Elemental Analysis System Using Conventional SEM- EDS
}

\author{
Shintaro Suzuki ${ }^{1}$, Masaki Morita ${ }^{1}$ and Yasunori Ohta ${ }^{1}$ \\ 1. JEOL Ltd. Tokyo, Japan.
}

Many users of SEM-EDS require automatic elemental analysis. The particle analysis system using SEMEDS is feasible to automatically analyze particles. Generally, particle analysis automatically identifies particles from the electron microscope image and carries out the elemental analysis by using EDS. In order to check the quality, steel companies have used the particle analysis to analyze the number and size of micron-sized inclusions in a metal. In recent years, there has been an increasing demand for analyzing submicron inclusions.

Analyzing submicron order particles by using SEM-EDS has an issue of the X-ray generation region. At an accelerating voltage of $15 \mathrm{kV}$, the $\mathrm{X}$-ray generation region is in the order of microns. It is necessary to set the accelerating voltage lower to decrease the X-ray generation region. However, at low accelerating voltage, the probe current decreases. In the particle analysis, analysis throughput is very important because the particle analysis analyzes many particles. Another important point is the accuracy of the probe position. The particle analysis must analyze a wide range in order to check the distribution of particles. The particle analysis is performed at low magnification and high resolution in order to reduce the number of images captured. At low magnification, the size of submicron order particles on the image is a few pixels. It is important that the probe can correctly irradiate these particles. To perform the particle analysis of submicron particles, the electron microscope must have a high-bright and stable probe current when operating at low accelerating voltages. The particle analysis system also requires the high throughput and the accuracy of the probe control.

We developed a particle analysis software having features of the high throughput and the high accuracy of the probe. By executing the acquisition of EDS and the analysis of X-ray spectrum in parallel, this software increases the throughput. We analyzed inclusions in steel by using combination of this particle analysis software and the high brightness conventional SEM "JSM-IT500HR" equipped with a 100 $\mathrm{mm}^{2}$ SDD. This SEM is suitable for the particle analysis by a thermal type field-emission electron gun and a large specimen chamber. The condition of the analysis was set to an acceleration voltage of $10 \mathrm{kV}$, magnification x600 times $(213 \times 160 \mu \mathrm{m})$ and probe current of about $3 \mathrm{nA}$. The measurement area was $1.28 \mathrm{~mm} \times 0.96 \mathrm{~mm}$ (36views), and target particle size for the measurement was more than $0.5 \mu \mathrm{m}$. EDS acquisition time was 0.1 seconds per particle. There were about one thousand particles in one view. This steel has $\mathrm{W}, \mathrm{SiO}_{2}$ and $\mathrm{MnS}$ inclusions. Figure 1 shows an example of an electron backscattering image of each inclusion and the corresponding X-ray spectrum.

The total analysis time, including image acquisition and stage movement time, was 2 hours 49 minutes for analyzing 40,421 particles in this steel. The throughput was 14,000 particles per hour. This throughput is very high in the particle analysis systems using conventional SEM-EDS. The accuracy of this particle analysis system was estimated from a number of identified particles and elemental analysis result. This particle analysis system correctly detected $99.8 \%$ particles exceeding $1 \mu \mathrm{m}$ size and $99.6 \%$ particles less than $1 \mu \mathrm{m}$ size. We confirmed that the combination of our particle analysis software and 
the conventional SEM-EDS enables us to analyze submicron order particles with high throughput and high accuracy.

References:

[1] Markus Nuspl et al, Anal Bioanal Chem (2004).
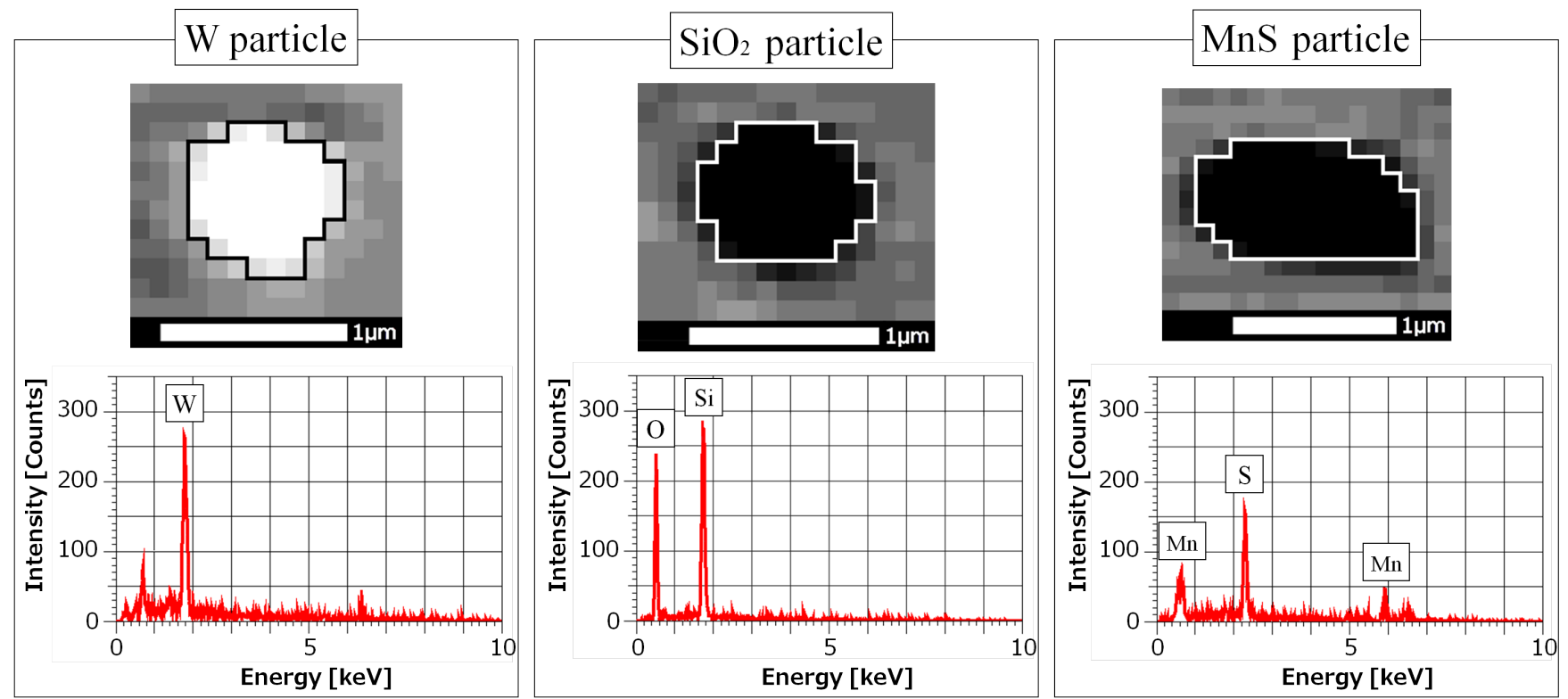

Figure 1. Example of electron backscattering image of each inclusion and its X-ray spectrum. 